Original research article

\title{
Nonsurgical lumbar radiculopathies treated with ultramicronized palmitoylethanolamide (UMPEA): A series of 100 cases
}

\author{
Domenico Chirchiglia ${ }^{a,}$, Pasquale Chirchiglia ${ }^{b}$, Francesco Signorelli $^{a}$ \\ a Department of Neurosurgery, University of Catanzaro, Campus Germaneto, Vle Europa, 88100 Catanzaro, Italy \\ ${ }^{\mathrm{b}}$ University of Catanzaro, Campus Germaneto, Vle Europa, 88100 Catanzaro, Italy
}

A R T I C L E I N F O

Article history:

Received 7 September 2017

Accepted 4 November 2017

Available online 14 November 2017

Keywords:

Palmitoylethanolamide

Low back pain

Degenerative spine diseases

Algometry

\section{Introduction}

Lumbar radiculopathy is a very frequent spine pathology. It afflicts people of all ages and it is mainly responsible for lower back pain, an often disabling symptom which in the end has a high impact in daily activities and in terms of economic costs. Causes are degenerative, regarding alterations of vertebral structures (both bone-cartilage and nerve-root), but may also be neoplastic, inflammatory, dismetabolic and other causes. Degenerative causes can be of surgical pertinence or not: surgical ones are mainly due to herniated discs and lumbar canal stenosis as well as spondylolisthesis. Generally, we refer to spondyloarthrosis, spondylo-discarthrosis, and disc protrusion, as terms to define all nonsurgical degenerative spine disorders in which lower back pain symptoms are prevalent, tending to become chronic for which the administration of pain relief drugs and physiotherapic support therapies can be helpful. One type of pharmacological therapy using micronized palmitoylethanolamide has long since been considered, exploiting the anti-inflammatory action of this compound on non-neuronal cells like mastcells and microglial neurons [1]. In this work we present a casuistry composed of nonsurgical lumbar radiculopathies treated with PEA and the relative obtained results.

\section{Materials and methods}

We analyzed retrospectively a number of 100 cases of patients with a long history of lower back pain with or without sciatica, composed of 73 males and 27 females, 45 and 75 years old,

\footnotetext{
* Corresponding author.

E-mail addresses: chirchiglia@unicz.it (D. Chirchiglia), solenero80@hotmail.it (P. Chirchiglia), fsignorelli@unicz.it (F. Signorelli). https://doi.org/10.1016/j.pjnns.2017.11.002 0028-3843/@ 2017 Polish Neurological Society. Published by Elsevier Sp. z o.o. All rights reserved.
} 
average age 60. Neurological examinations showed signs of lumbar radicular compression such as reduction or abolition of tendon reflexes, decrease in muscle strength, in some cases so severe to force the patient to stay in bed. Radiological diagnostic (Radiography) procedures showed more or less marked signs of spondylosis in all patients and CT scans and spine MRI confirmed vertebral bone alterations, as well as modifications in all structures like intervertebral disc, constituting by black disc, disc protrusion, and dehydration disc. Laboratory results were in the standard. Furthermore, all subjects underwent diagnostic neurophysiological assessment (Needle EMG, nerve conduction velocities), which showed electrophysiological signs of radicular ache in less than half of cases. Then, the patients underwent pain evaluation, using Visual Analogic Scale (VAS) that showed a score between 8 and $10 \mathrm{~cm}$ in 72 subjects, $5-6 \mathrm{~cm}$ in 14, 2-4 cm in the fourteen remaining. On the basis of these results, the radicular compression was divided into mild, moderate and severe. At this point, a pharmacological protocol was prescribed, consisting in administration of um-PEA, formulated as follows: ultramicronized form $1200 \mathrm{mg} /$ die, sachet confection sublingual use for 10 days, then the same dose in tablets for the remaining 20 days (Normast ${ }^{\circledR} 600 \mathrm{mg}$ microgranules and tablets; Epitech Group S.p.A.-Saccolongo, Italy). The second cycle was for the duration of another month and consisted in administration of ultramicronized form $600 \mathrm{mg} /$ die, tablet use. In combination with PEA, in both cycles, paracetamol $500 \mathrm{mg}$ plus codeine $30 \mathrm{mg} /$ die for 4 days was used, then only as needed, for 1 month; paracetamol combined with codeine was used for the second month, at the same dose only as needed.

\section{Results}

Results have been as follows: after 1 month of therapy, the patients with baseline score mild (VAS between 2 and 4; 14 patients), moderate (VAS between 5 and 6; 14 patients) and severe (VAS between 8 and 10; 72 patients) showed a significant $(P<0.0001)$ decrease of average pain intensity respectively of $1.9 \pm 0.10,2.7 \pm 0.13$ and $2.3 \pm 0.19$. After the second cycle of therapy, all patients reported a further improvement in pain, as pointed out by VAS, particularly those with a mild and moderate score were pain free (Figs. 1 and 2), while the $70.80 \%$ of patients considered as severe score showed an improvement of more than $50 \%$ (Fig. 3). Nobody during the two cycles of therapy reported any adverse effects. Therefore, the results offered by clinical and pharmacological data show the effective efficacy of PEA in improving pain symptomatology in degenerative lumbar radicular compression, as noted in our report of cases with a total success percentage of about $80 \%$, with a greater effect on those with mild and moderate grade of pain.

\section{Discussion}

Palmitoylethanolamide (PEA) is an endogenous chemical substance of lipid nature, identified in the 1990s by the research group of Nobel Laureate Rita Levi Montalcini as regulator of mastcell reactivity [2]. It acts on not only neuronal cells but mastcells and microglial cells as well, modulating microglial activation and mastcell hyper-reactivity, therefore having a role in neuroinflammation events (ALIA mechanism: Autacoid Local Injury Antagonism) [2,3]. The pathophysiology of neuroinflammation starts from the interaction between nociceptive terminations and mastcells. A low control on mastcell provokes abnormal release of growth factors (with neo-innervation, angiogenesis), biogenic amines (with development of edema), cytokines, causing reduct algogenic threshold (peripheral sensitization) and neurogenic inflammation [4,5]. PEA intervenes in this mechanism, when esogenous provision is necessary, blocking spinal neuroinflammation processes and neuronal plasticity which cause marked and amplified modifications of synaptic transmission [6]. It is the failure to control this that creates and keeps neuropathic pain, for which the intervention of PEA is helpful and necessary. Furthermore, PEA normalizes mastcell de-

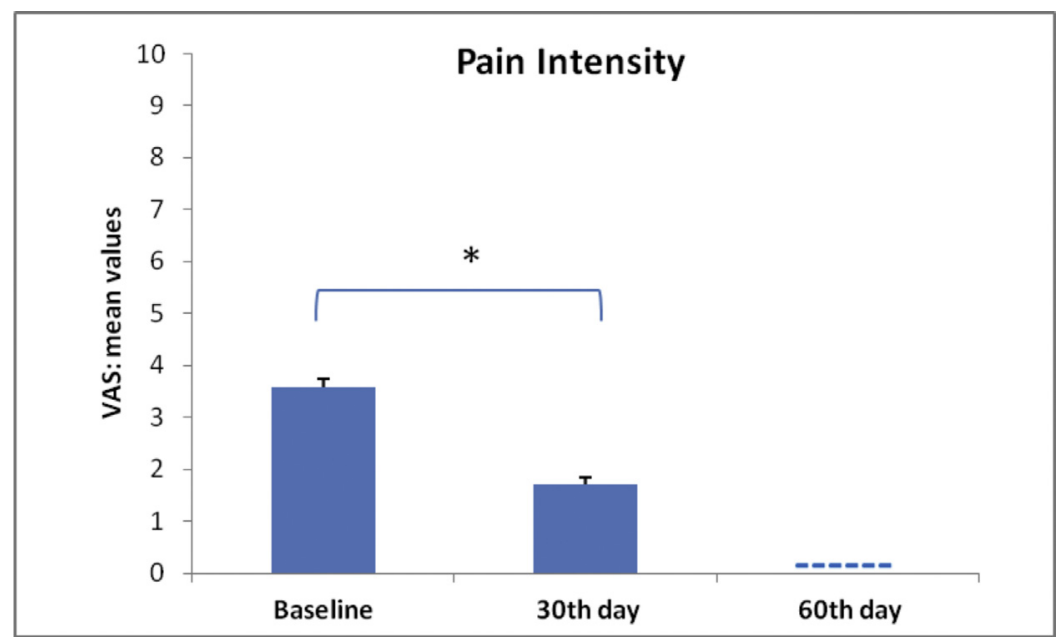

Fig. 1 - Decrease over time of pain intensity observed in 14 patients suffering from mild painful symptoms (baseline score between 2 and 4; the mean values move from $3.5 \pm 0.2$ to $1.7 \pm 0.1$ and to 0 ). ${ }^{*} P<0.0001$. 


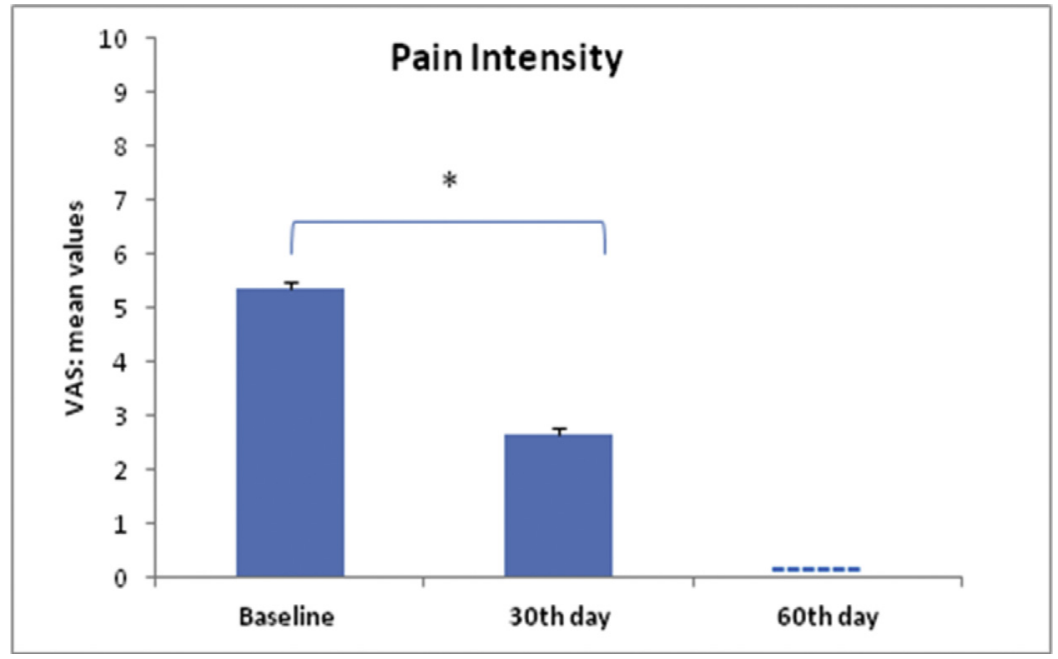

Fig. 2 - Decrease over time of pain intensity observed in 14 patients suffering from moderate painful symptoms with baseline score between 5 and 6 ; the mean values move from $5.3 \pm 0.1$ to $2.6 \pm 0.1$ and to $0 .{ }^{*} P<0.0001$.

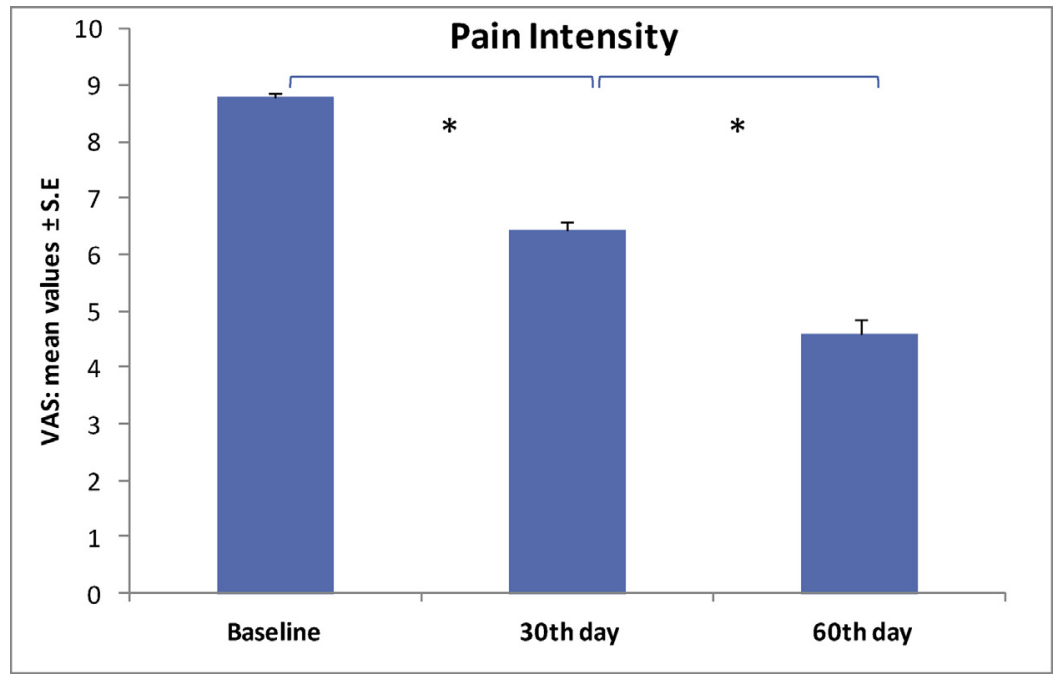

Fig. 3 - Decrease over time of pain intensity observed in 72 patients, suffering from severe painful symptoms with baseline score between 8 and 10 ; the mean values move from $8.7 \pm 0.10$ to $6.4 \pm 0.15$ and to $4.6 \pm 0.29$. ${ }^{*} P<0.0001$.

granulation and microglial activation thereby maintaining tissue homeostasys and therefore in a progressive decrease in endogenous production of PEA leads to an inability to regulate mastcells and microglial cells, creating pain symptoms $[7,8]$. Therefore um-PEA (Ultramicronized Palmithoylethanolamide) is used in those diseases in which the mechanisms of neuroinflammation are present such as neuropathies, by nerve entrapment [9] or dismetabolic [10], toxic [11] and others [12-15] and some primary headaches [16]. In this study, we used um-PEA in degenerative lumbar radiculopathies, a very frequent disorder, related to alterations involving the various structures of the lumbar spine, as vertebral body, intervertebral disc and ligament, which, at the end, cause root compression and consequently onset of pain. Edema is an associated condition, worsening the pain and is a target of umPEA pharmacological action, through previously aforementioned mechanisms. Furthermore, these diseases become chronic and without surgical intervention the patient has to live with the pain and take pain relieving drugs such as FANS and steroids, besides physiotherapic treatments. Our study shows satisfying results in patients treated with um-PEA, with an excellent percentage of success, mostly regarding mild and moderate patients, who appeared as the best responders to um-PEA therapy. It encourages us to continue the studies on um-PEA in order to better establish and improve the treatment and so offer a better quality of life to these patients. 


\section{Conclusions}

This work demonstrates the effectiveness and safety of umPEA in lumbar radicular compression, envisaging interesting future perspectives in pain treatment. Our described cases showed a good response to therapy, as highlighted by two months clinical controls, estimated at $80 \%$ of patients tested, mainly in those with mild and moderate chronic pain, which, at the end of control study, reported no lower back pain or sciatica. Another aspect worthy of note is the total absence of side effects. In conclusion, our study, utilizing a discreet number of patients, showed positive results in lumbar radiculopathies, in both conditions, pain and quality of life, in nonsurgical subjects.

\section{Conflict of interest}

None declared.

\section{Acknowledgment and financial support}

None declared.

\section{REFEREN CES}

[1] Guida G, de Fabiani A, Lanaia F, Alexandre A, Vassallo GM, Cantieri L, et al. La palmitoiletanolamida (Normast ${ }^{\circledR}$ ) en el dolor neuropático crónico por lumbociatalgia de tipo compresivo: estudio clínico multicéntrico. Dolor 2010;25:35-42.

[2] Facci L, Dal Toso R, Romanello S, Buriani A, Skaper SD, Leon A. Mast cells express a peripheral cannabinoid receptor with differential sensitivity to anandamide and Palmitoylethanolamide. Proc Nati Acad Sci 1995;92:3376-80.

[3] Levi-Montalcini R, Skaper SD, Dal Toso R, Petrelli L, Leon A. Nerve growth factor: from neurotrophin to neurokine. Trends Neurosci 1996;19(11):514-20.

[4] Bettoni I, Comelli F, Colombo A, Bonfanti P, Costa B. Nonneuronal cell modulation relieves neuropathic pain: efficacy of the endogenous lipid palmitoylethanolamide. CNS Neurol Disord Drug Targets 2013;12(1):34-44.
[5] Skaper SD, Facci L, Fusco M, Della Valle MF, Zusso M, Costa $\mathrm{B}$, et al. Palmitoylethanolamide, a naturally occurring disease-modifying agent in neuropathic pain. Inflammopharmacology 2014;22(2):79-94.

[6] Skaper SD, Giusti P, Facci L. Microglia and mast cells: two tracks on the road to neuroinflammation. FASEB J 2012;26 (8):3103-17.

[7] Skaper SD, Facci L. Mast cell - glia axis in neuroinflammation and therapeutic potential of the anandamide congener palmitoylethanolamide. Philos Trans R Soc Lond B Biol Sci 2012;367(1607):3312-25.

[8] Cocito D, Peci E, Ciaramitaro P, Merola A, Lopiano L. Shortterm efficacy of ultramicronized palmitoylethanolamide in peripheral neuropathic pain. Pain Res Treat 2014;2014:854560. http://dx.doi.org/10.1155/2014/854560

[9] Conigliaro R, Drago V, Foster PS, Schievano C, Di Marzo V. Use of palmitoylethanolamide in the entrapment neuropathy of the median in the wrist. Minerva Medica 2011;102(2):141-7.

[10] Schifilliti C, Cucinotta L, Fedele V, Ingegnosi C, Luca S, Leotta C. Micronized palmitoylethanolamide reduces the symptoms of neuropathic pain in diabetic patients. Pain Res Treat 2014;2014:849623.

[11] Truini A, Biasiotta A, Di Stefano G, La Cesa S, Leone C, Cartoni C, et al. Palmitoylethanolamide restores myelinated-fibre function in patients with chemotherapyinduced painful neuropathy. CNS Neurol Disord Drug Targets 2011;10(8):916-20.

[12] Gatti A, Lazzari M, Gianfelice V, Di Paolo A, Sabato E, Sabato AF. Palmitoylethanolamide in the treatment of chronic pain caused by different etiopathogeneses. Pain Med 2012;13 (9):1121-30

[13] Paladini A, Fusco M, Cenacchi T, Schievano C, Piroli A, Varrassi G. Palmitoylethanolamide, a special food for medical purposes, in the treatment of chronic pain: a pooled data meta-analysis. Pain Physician 2016;19(2):11-24.

[14] Del Giorno R, Skaper S, Paladini A, Varrassi G, Coaccioli S. Palmitoylethanolamide in fibromyalgia: results from prospective and retrospective observational studies. Pain Ther 2015;4(2):169-78.

[15] Paladini A, Varrassi G, Bentivegna G, Carletti S, Piroli A, Coaccioli S. Palmitoylethanolamide in the treatment of failed back surgery syndrome. Pain Res Treat 2017. http:// dx.doi.org/10.1155/2017/1486010

[16] Chirchiglia D, Della Torre A, Signorelli F, Volpentesta G, Guzzi G, Stroscio CA, et al. Administration of palmitoylethanolamide in combination with topiramate in the preventive treatment of nummular headache. Int Med Case Rep J 2016;9:193-5. 